\title{
Research Article \\ Weak Convergence of Markov Random Evolutions in a Multidimensional Space
}

\author{
Igor V. Samoilenko \\ Institute of Mathematics, NASU, Tereschenkivska Street, 3, Kiev 01601, Ukraine \\ Correspondence should be addressed to Igor V. Samoilenko, isamoil@imath.kiev.ua
}

Received 4 May 2012; Accepted 5 June 2012

Academic Editors: E. Orsingher and A. Pascucci

Copyright (C) 2012 Igor V. Samoilenko. This is an open access article distributed under the Creative Commons Attribution License, which permits unrestricted use, distribution, and reproduction in any medium, provided the original work is properly cited.

We study Markov symmetrical and nonsymmetrical random evolutions in $\mathbf{R}^{n}$. Weak convergence of Markov symmetrical random evolution to Wiener process and of Markov non-symmetrical random evolution to a diffusion process with drift is proved using problems of singular perturbation for the generators of evolutions. Relative compactness in $\mathbf{D}_{\mathbf{R}^{n} \times \Theta}[0, \infty)$ of the families of Markov random evolutions is also shown.

\section{Introduction}

Markov symmetrical random evolutions (MSRE) in spaces of different dimensions were studied in the works of Kac [1], Pinsky [2], Orsingher (e.g., [3, 4]), and Kolesnik and Turbin (e.g., [5, 6]) (see also [7] for other references). Symmetry in this sense should be regarded as uniform stationary distribution of switching at a symmetrical structure in $\mathbf{R}^{n}$, for instance at $n+1$-hedron [7], or at a unit sphere [8].

Weak convergence of distributions of MSRE is also studied in some of these works, namely, convergence in $\mathbf{R}^{2}$ and $\mathbf{R}^{3}$ was proved by Kolesnik in $[8,9]$.

In this work we not only generalize the results of Kolesnik on the multidimensional space, but solve another problem. We prove weak convergence of the process of Markov random evolution, that means not only proof of weak convergence of respective distributions (or generators) but also proof of compactness of the process. In majority of works that deal with random evolutions, compactness of the process is not considered at all. It is also shown that the symmetry of the process is closely connected with balance condition (see (2.23), (4.4)).

We should note that the problem of weak convergence of random walks (partially, similar to MSRE) was studied by many authors. Among the most interesting works, we can point out [10-13]. Large bibliography as for this problem could be found in [10]. The 
methods proposed in these works allow us to solve a wide range of problems connected with convergence of random walks but do not let us obtain limit process to be averaged by the stationary measure of switching process.

Such averaging can be found in the works of Anisimov and his students (see [14] and references therein), but here the averaging by the stationary measure is one of the conditions proposed for the prelimit process in the corresponding theorem.

In this work, MSRE in $\mathbf{R}^{n}$ is studied using the methods proposed in [15]. We find a solution of singular perturbation problem for the generator of the evolution, and thus the averaging by a stationary measure of switching process is obtained as a corollary of this solution. At the second stage we prove relative compactness of the family of MSRE. This method let us show weak convergence of the process of MSRE to the Wiener process in $\mathbf{R}^{n}$.

The difference in the methods can be easily seen by the analysis of the papers $[16,17]$, where similar problems are studied.

In Sections 3 and 4, we use the method proposed to prove weak convergence of Markov non-symmetrical random evolution (MNRE) in $\mathbf{R}^{n}$. The distinction of this model is that the limit process is a diffusion process with drift.

\section{Description of MSRE}

We study a particle in the space $\mathbf{R}^{n}$, that starts at $t=0$ from the point $x=\left(x_{i}, i=\overline{1, n}\right)$. Possible directions of motion are given by the following vectors:

$$
\begin{array}{r}
s(\theta)=\left(\cos \theta_{1}, \sin \theta_{1} \cos \theta_{2}, \sin \theta_{1} \sin \theta_{2} \cos \theta_{3}, \ldots, \sin \theta_{1} \cdots \sin \theta_{n-2} \cos \theta_{n-1},\right. \\
\left.\sin \theta_{1} \cdots \sin \theta_{n-2} \sin \theta_{n-1}\right), \quad \theta_{n-1} \in[0,2 \pi), \theta_{i} \in[0, \pi), i=\overline{1, n-2} .
\end{array}
$$

These vectors have initial point in the center of the unit $n$-dimentional sphere $S_{n}$ and the terminal point at its surface. Choosing of every next direction is random and its time is distributed by the Poisson law. Thus, the switching process process is the Poisson one with intensity $\lambda=\varepsilon^{-2}$. The velocity of particle's motion is fixed and equals $v=c \varepsilon^{-1}$, where $\varepsilon$ is a small parameter, $\varepsilon \rightarrow 0(\varepsilon>0)$.

Let us define a set $\Theta=\left\{\theta: s(\theta) \in S_{n}\right\}$ and suppose $\theta_{t}^{\varepsilon} \in \Theta$ be the switching Poisson process.

Definition 2.1. Markov symmetrical random evolution (MSRE) is the process $\xi_{t}^{\varepsilon} \in \mathbf{R}^{n}$, given by

$$
\xi_{t}^{\varepsilon}:=x+v \int_{0}^{t} s\left(\theta_{\tau}^{\varepsilon}\right) d \tau
$$

It is easy to see that when $\varepsilon \rightarrow 0(\varepsilon>0)$, the velocity of the particle and intensity of switching decrease. Our aim is to prove weak convergence of MSRE to the Wiener process when $\varepsilon \rightarrow 0$. The main method is solution of singular perturbation problem for the generator of MSRE. Let us describe this generator. 
Two-component Markov process $\left(\xi_{t}^{\varepsilon}, \theta_{t}^{\varepsilon}\right)$ at the test functions $\varphi\left(x_{1}, \ldots, x_{n} ; \theta\right) \in C_{0}^{\infty}\left(\mathbf{R}^{n} \times\right.$ $\Theta)$ can be described by the generator (see, e.g., [2])

$$
\begin{aligned}
L^{\varepsilon} \varphi\left(x_{1}, \ldots, x_{n} ; \theta\right) & :=\lambda Q \varphi(\cdot ; \theta)+v S(\theta) \varphi\left(x_{1}, \ldots, x_{n} ; \cdot\right) \\
& =\varepsilon^{-2} Q \varphi(\cdot ; \theta)+\varepsilon^{-1} c S(\theta) \varphi\left(x_{1}, \ldots, x_{n} ; \cdot\right)
\end{aligned}
$$

where

$$
S(\theta) \varphi\left(x_{1}, \ldots, x_{n} ; \cdot\right):=-(s(\theta), \nabla) \varphi\left(x_{1}, \ldots, x_{n} ; \cdot\right)
$$

here $\nabla \varphi=\left(\partial \varphi / \partial x_{i}, 1 \leq i \leq n\right)$,

$$
Q \varphi(\cdot ; \theta):=(\Pi-I) \varphi(\cdot ; \theta):=\frac{1}{N} \int_{S_{n}} \varphi(\cdot ; \theta) \mu(d \theta)-\varphi(\cdot ; \theta),
$$

and $N=(2 \pi)^{n / 2}(1 /(2 \cdot 4 \cdots(n-2)))$ for even $n, N=(2 \pi)^{(n-1) / 2}(2 /(3 \cdot 5 \cdots \cdots(n-2)))$ for odd $n ; \mu(d \theta)$ is the element of volume of the sphere $S_{n}$, that is equal to

$$
\mu(d \theta):=\sin ^{n-2} \theta_{1} \sin ^{n-3} \theta_{2} \cdots \sin \theta_{n-2} d \theta_{1} \cdots d \theta_{n-1} .
$$

Using well-known formula

$$
\begin{aligned}
& \int_{0}^{\pi} \sin ^{2 m} d \theta=2 \cdot \frac{1 \cdot 2 \cdots(2 m-1)}{2 \cdot 4 \cdots 2 m} \cdot \frac{\pi}{2} \\
& \int_{0}^{\pi} \sin ^{2 m+1} d \theta=2 \cdot \frac{2 \cdot 4 \cdots 2 m}{1 \cdot 3 \cdots \cdots(2 m+1)}
\end{aligned}
$$

we can see

$$
\int_{S_{n}} \mu(d \theta)=N
$$

Operator $\Pi \varphi(\cdot ; \theta):=(1 / N) \int_{S_{n}} \varphi(\cdot ; \theta) \mu(d \theta)$ is the projector at the null space of reducibly invertible operator $Q$, because by definition it maps functions to constants but constants to itself.

For $\Pi$ we have

$$
Q \Pi=\Pi Q=0 .
$$

Potential operator $R_{0}$ can be defined as the following:

$$
R_{0}:=\Pi-I
$$


This operator has the following property:

$$
R_{0} Q=Q R_{0}=I-\Pi
$$

thus it is inverse for $Q$ at the range of $Q$, but for the function $\phi$ from the null space of $Q$ we have

$$
R_{0} \phi=0
$$

Solution of singular perturbation problem in the series scheme with the small series parameter $\varepsilon \rightarrow 0(\varepsilon>0)$ (see [15]) for reducibly invertible operator $Q$ and perturbing operator $Q_{1}$ consists in the following.

We should find two vectors $\varphi^{\varepsilon}=\varphi+\varepsilon \varphi_{1}+\varepsilon^{2} \varphi_{2}$ and $\psi$, that satisfy asymptotic representation

$$
\left[\varepsilon^{-2} Q+\varepsilon^{-1} Q_{1}\right] \varphi^{\varepsilon}=\psi+\varepsilon \theta^{\varepsilon},
$$

with the vector $\theta^{\varepsilon}$, that is uniformly bounded by the norm and such that

$$
\left\|\theta^{\varepsilon}\right\| \leq C, \quad \varepsilon \longrightarrow 0 .
$$

The left part of the equation can be rewritten as

$$
\begin{aligned}
{\left[\varepsilon^{-2} Q\right.} & \left.+\varepsilon^{-1} Q_{1}\right]\left(\varphi+\varepsilon \varphi_{1}+\varepsilon^{2} \varphi_{2}\right) \\
& =\varepsilon^{-2} Q \varphi+\varepsilon^{-1}\left[Q \varphi_{1}+Q_{1} \varphi\right]+\left[Q \varphi_{2}+Q_{1} \varphi_{1}\right]+\varepsilon Q_{1} \varphi_{2} .
\end{aligned}
$$

And as soon as it is equal to the right side, we obtain

$$
\begin{gathered}
Q \varphi=0, \\
Q \varphi_{1}+Q_{1} \varphi=0, \\
Q \varphi_{2}+Q_{1} \varphi_{1}=\psi, \\
Q_{1} \varphi_{2}=\theta^{\varepsilon} .
\end{gathered}
$$

From the last equation we may see that the function $\varphi_{2}$ should be smooth enough to provide boundness of $Q_{1} \varphi_{2}$. Moreover, from the first equation we see that any function from the null space of $Q$ can be taken as $\varphi$, and it does not depend on the variable that corresponds to the switching process.

An important condition of solvability for this problem is the balance condition

$$
\Pi Q_{1}=0
$$


This condition means that the function $Q_{1} \varphi$ belongs to the range of $Q$, thus we may solve the second equation using the potential operator, that is, inverse to $Q$ at its range

$$
\varphi_{1}=-R_{0} Q_{1} \varphi
$$

Thus, the main problem is to solve the following equation:

$$
Q \varphi_{2}=\psi-Q_{1} \varphi_{1}=\psi+Q_{1} R_{0} Q_{1} \varphi
$$

The solvability condition for $Q$ has the view

$$
\Pi Q \Pi \varphi_{2}=0=\Pi \psi+\Pi Q_{1} R_{0} Q_{1} \Pi \varphi
$$

and we finally obtain

$$
\Pi \psi=-\Pi Q_{1} R_{0} Q_{1} \Pi \varphi
$$

For the function $\varphi_{2}$ we obviously have

$$
\varphi_{2}=R_{0}\left[-\Pi Q_{1} R_{0} Q_{1} \Pi+Q_{1} R_{0} Q_{1}\right] \varphi
$$

Equations (2.18)-(2.22) give the solution of singular perturbation problem.

In case of MSRE, the balance condition has the following view:

$$
\Pi S(\theta) \mathbf{1}(x)=0,
$$

where $\mathbf{1}(x)=\left(x_{1}, \ldots, x_{n}\right)$. Really, every term under the integral contains either $\int_{0}^{\pi} \sin ^{n} \theta \cos \theta d \theta=0$ or $\int_{0}^{2 \pi} \sin \theta d \theta=0$.

\section{Main Result for MSRE}

Theorem 3.1. MSRE $\xi_{t}^{\varepsilon}$, converges weakly to the Wiener process $w(t):=\xi_{t}^{0}$ when $\varepsilon \rightarrow 0$ as

$$
\xi_{t}^{\varepsilon} \Longrightarrow \xi_{t}^{0}
$$

where $\xi_{t}^{0} \in \mathbf{R}^{n}$ is defined by the generator

$$
L^{0} \varphi\left(x_{1}, \ldots, x_{n}\right)=\frac{c^{2}}{n} \Delta \varphi\left(x_{1}, \ldots, x_{n}\right) .
$$

Here $\Delta \varphi:=\left(\left(\partial^{2} /\left(\partial x_{1}^{2}\right)\right)+\cdots+\left(\partial^{2} /\left(\partial x_{n}^{2}\right)\right)\right) \varphi$.

Remark 3.2. The generator (3.2) generalizes the generators obtained in [8] for the spaces $\mathbf{R}^{2}$ and $\mathbf{R}^{3}$.

We use the following Lemma to prove the Theorem. 
Lemma 3.3. At the perturbed test functions

$$
\varphi^{\varepsilon}\left(x_{1}, \ldots, x_{n} ; \theta\right)=\varphi\left(x_{1}, \ldots, x_{n}\right)+\varepsilon \varphi_{1}\left(x_{1}, \ldots, x_{n} ; \theta\right)+\varepsilon^{2} \varphi_{2}\left(x_{1}, \ldots, x_{n} ; \theta\right),
$$

having bounded derivatives of any degree and compact support, the operator $L^{\varepsilon}$ has the asymptotic representation

$$
\begin{gathered}
L^{\varepsilon} \varphi^{\varepsilon}\left(x_{1}, \ldots, x_{n} ; \theta\right)=L^{0} \varphi\left(x_{1}, \ldots, x_{n}\right)+R^{\varepsilon}(\theta) \varphi(x), \\
\left|R^{\varepsilon}(\theta) \varphi(x)\right| \longrightarrow 0, \quad \varepsilon \longrightarrow 0, \quad \varphi(x) \in C_{0}^{\infty}\left(\mathbf{R}^{n}\right),
\end{gathered}
$$

where $L^{0}$ is defined in (3.2), $\varphi_{1}\left(x_{1}, \ldots, x_{n} ; \theta\right), \varphi_{2}\left(x_{1}, \ldots, x_{n} ; \theta\right)$ and $R^{\varepsilon}(\theta) \varphi(x)$ are the following:

$$
\begin{gathered}
L^{0} \Pi=-c^{2} \Pi S(\theta) R_{0} S(\theta) \Pi, \\
\varphi_{1}=-c R_{0} S(\theta) \varphi, \\
\varphi_{2}=c^{2} R_{0} S(\theta) R_{0} S(\theta) \varphi, \\
R^{\varepsilon}(\theta) \varphi=\varepsilon c^{3} S(\theta) R_{0} S(\theta) R_{0} S(\theta) \varphi .
\end{gathered}
$$

Proof. Let us solve singular perturbation problem for the operator (2.3). To do this, we use the following correlation:

$$
\begin{aligned}
L^{\varepsilon} \varphi^{\varepsilon}\left(x_{1}, \ldots, x_{n} ; \theta\right) & =\left[\varepsilon^{-2} Q+\varepsilon^{-1} c S(\theta)\right]\left[\varphi+\varepsilon \varphi_{1}+\varepsilon^{2} \varphi_{2}\right] \\
& =\varepsilon^{-2} Q \varphi+\varepsilon^{-1}\left[Q \varphi_{1}+c S(\theta) \varphi\right]+\left[Q \varphi_{2}+c S(\theta) \varphi_{1}\right]+\varepsilon c S(\theta) \varphi_{2} .
\end{aligned}
$$

Thus, we obtain equations

$$
\begin{gathered}
Q \varphi=0, \\
Q \varphi_{1}+c S(\theta) \varphi=0, \\
L^{0} \varphi=Q \varphi_{2}+c S(\theta) \varphi_{1}, \\
R^{\varepsilon} \varphi(\theta)=\varepsilon c S(\theta) \varphi_{2} .
\end{gathered}
$$

From the first equation we see that $\varphi\left(x_{1}, \ldots, x_{n}\right)$ belongs to the null space of $Q$. It is easy to see from the balance condition (2.23) that $S(\theta) \varphi$ belongs to the range of $Q$, thus from the second equation of the system (3.7) we have

$$
\varphi_{1}=-c R_{0} S(\theta) \varphi
$$

By substitution into the third equation and using the solvability condition, we can see

$$
L^{0} \Pi \varphi+c^{2} \Pi S(\theta) R_{0} S(\theta) \Pi \varphi=0 .
$$


ISRN Probability and Statistics

From the last equation of (2.23) we have

$$
R^{\varepsilon}(\theta) \varphi(x)=\varepsilon c^{3} S(\theta) R_{0} S(\theta) R_{0} S(\theta) \varphi(x) \longrightarrow 0 \quad \text { when } \varepsilon \longrightarrow 0, \varphi(x) \in C_{0}^{\infty}\left(\mathbf{R}^{n}\right)
$$

Let's find the generator of the limit process $L^{0}$ by the formula (3.5) as

$$
L^{0} \varphi=c^{2} \Pi S(\theta)(I-\Pi) S(\theta) \Pi \varphi=c^{2} \Pi S^{2}(\theta) \Pi \varphi-c^{2} \Pi S(\theta) \Pi S(\theta) \Pi \varphi .
$$

The last term equals to 0 by the balance condition (2.23). Thus, finally

$$
L^{0}=c^{2} \Pi S^{2}(\theta)
$$

or

$$
L^{0}=\frac{c^{2}}{N} \int_{S_{n}} S^{2}(\theta) \mu(d \theta)
$$

Let us calculate the following integral:

$$
\begin{aligned}
& \frac{c^{2}}{N} \int_{0}^{\pi} \cdots \int_{0}^{\pi} \int_{0}^{2 \pi}\left[\cos ^{2} \theta_{1} \frac{\partial^{2}}{\partial x_{1}^{2}}+\sin ^{2} \theta_{1} \cos ^{2} \theta_{2} \frac{\partial^{2}}{\partial x_{2}^{2}}+\cdots+\sin ^{2} \theta_{1} \cdots \sin ^{2} \theta_{n-2} \cos ^{2} \theta_{n-1} \frac{\partial^{2}}{\partial x_{n-1}^{2}}\right. \\
& +\sin ^{2} \theta_{1} \cdots \sin ^{2} \theta_{n-2} \sin ^{2} \theta_{n-1} \frac{\partial^{2}}{\partial x_{n}^{2}} \\
& +\left\{\sin \theta_{1} \cos \theta_{1} \cos \theta_{2} \frac{\partial^{2}}{\partial x_{1} \partial x_{2}}+\cdots+\sin ^{2} \theta_{1} \cdots \sin ^{2} \theta_{n-2} \sin \theta_{n-1}\right. \\
& \left.\left.\times \cos \theta_{n-1} \frac{\partial^{2}}{\partial x_{n-1} \partial x_{n}}\right\}\right] \\
& \times \sin ^{n-2} \theta_{1} \sin ^{n-3} \theta_{2} \cdots \sin \theta_{n-2} d \theta_{1} \cdots d \theta_{n-1} \\
& =\frac{c^{2}}{N} \int_{0}^{\pi} \cdots \int_{0}^{\pi} \int_{0}^{2 \pi}\left[\cos ^{2} \theta_{1} \sin ^{n-2} \theta_{1} \sin ^{n-3} \theta_{2} \cdots \sin \theta_{n-2} \frac{\partial^{2}}{\partial x_{1}^{2}}\right. \\
& +\sin ^{n} \theta_{1} \cos ^{2} \theta_{2} \sin ^{n-3} \theta_{2} \cdots \sin \theta_{n-2} \frac{\partial^{2}}{\partial x_{2}^{2}} \\
& +\cdots+\sin ^{n} \theta_{1} \sin ^{n-1} \theta_{2} \cdots \sin ^{3} \theta_{n-2} \cos ^{2} \theta_{n-1} \\
& \times \frac{\partial^{2}}{\partial x_{n-1}^{2}}+\sin ^{n} \theta_{1} \sin ^{n-1} \theta_{2} \cdots \sin ^{2} \theta_{n-2} \sin ^{2} \theta_{n-1} \frac{\partial^{2}}{\partial x_{n}^{2}}
\end{aligned}
$$




$$
\begin{aligned}
& +\left\{\sin ^{n-1} \theta_{1} \cos \theta_{1} \cos \theta_{2} \sin ^{n-3} \theta_{2} \cdots \sin \theta_{n-2} \frac{\partial^{2}}{\partial x_{1} \partial x_{2}}\right. \\
& \left.\left.+\cdots+\sin ^{n} \theta_{1} \sin ^{n-1} \theta_{2} \cdots \sin ^{3} \theta_{n-2} \sin \theta_{n-1} \cos \theta_{n-1} \frac{\partial^{2}}{\partial x_{n-1} \partial x_{n}}\right\}\right] \\
& \times d \theta_{1} \ldots d \theta_{n-1} \\
& =\frac{c^{2}}{N} \int_{0}^{\pi} \int_{0}^{\pi} \cdots \int_{0}^{\pi}\left[\left(\sin ^{n-2} \theta_{1} \sin ^{n-3} \theta_{2} \cdots \sin \theta_{n-2}\right.\right. \\
& \left.-\sin ^{n} \theta_{1} \sin ^{n-3} \theta_{2} \cdots \sin \theta_{n-2}\right) \frac{\partial^{2}}{\partial x_{1}^{2}} \\
& +\left(\sin ^{n} \theta_{1} \sin ^{n-3} \theta_{2} \cdots \sin \theta_{n-2}\right. \\
& \left.-\sin ^{n} \theta_{1} \sin ^{n-1} \theta_{2} \sin ^{n-4} \theta_{3} \cdots \sin \theta_{n-2}\right) \\
& \times \frac{\partial^{2}}{\partial x_{2}^{2}}+\cdots+\left(\sin ^{n} \theta_{1} \sin ^{n-1} \theta_{2} \cdots \sin ^{3} \theta_{n-2}\right. \\
& \left.-\sin ^{n} \theta_{1} \sin ^{n-1} \theta_{2} \cdots \sin ^{3} \theta_{n-2} \sin ^{2} \theta_{n-1}\right) \frac{\partial^{2}}{\partial x_{n-1}^{2}} \\
& +\sin ^{n} \theta_{1} \sin ^{n-1} \theta_{2} \cdots \sin ^{3} \theta_{n-2} \sin ^{2} \theta_{n-1} \frac{\partial^{2}}{\partial x_{n}^{2}} \\
& +\left\{\sin ^{n-1} \theta_{1} \cos \theta_{1} \cos \theta_{2} \sin ^{n-3} \theta_{2} \cdots \sin \theta_{n-2} \frac{\partial^{2}}{\partial x_{1} \partial x_{2}}\right. \\
& \left.\left.+\cdots+\sin ^{n} \theta_{1} \sin ^{n-1} \theta_{2} \cdots \sin ^{3} \theta_{n-2} \sin \theta_{n-1} \cos \theta_{n-1} \frac{\partial^{2}}{\partial x_{n-1} \partial x_{n}}\right\}\right] \\
& \times d \theta_{1} \cdots d \theta_{n-1} .
\end{aligned}
$$

Every term in braces has a multiplier of the type $\int_{0}^{\pi} \sin ^{n} \theta \cos \theta d \theta=0$ or $\int_{0}^{2 \pi} \sin \theta \cos \theta d \theta=0$, thus the corresponding integral equals to 0 .

Integration of the correlations in the parentheses gives

$$
\begin{aligned}
& \int_{0}^{\pi} \cdots \int_{0}^{\pi} \int_{0}^{2 \pi}\left(\sin ^{n} \theta_{1} \sin ^{n-1} \theta_{2} \cdots \sin ^{n-k+2} \theta_{k-1} \sin ^{n-k-1} \theta_{k} \sin ^{n-k-2} \theta_{k+1} \cdots \sin \theta_{n-2}\right. \\
& \left.\quad-\sin ^{n} \theta_{1} \sin ^{n-1} \theta_{2} \cdots \sin ^{n-k+2} \theta_{k-1} \sin ^{n-k+1} \theta_{k} \sin ^{n-k-2} \theta_{k+1} \cdots \sin \theta_{n-2}\right) d \theta_{1} \cdots d \theta_{n-1} \\
& =N\left(\frac{(2 m-1)(2 m-2)(2 m-3) \cdots(2 m-k+1)}{2 m(2 m-1)(2 m-2) \cdots(2 m-k+2)}\right.
\end{aligned}
$$




$$
\begin{aligned}
& \left.\quad-\frac{(2 m-1)(2 m-2)(2 m-3) \cdots(2 m-k)}{2 m(2 m-1)(2 m-2) \cdots(2 m-k+1)}\right) \\
& =N\left(\frac{2 m-k+1}{2 m}-\frac{2 m-k}{2 m}\right), \quad \text { if } n=2 m \\
& \text { or } N\left(\frac{(2 m)(2 m-1)(2 m-2) \cdots(2 m-k+2)}{(2 m+1) 2 m(2 m-1) \cdots(2 m-k+3)}\right. \\
& \left.\quad-\frac{2 m(2 m-1)(2 m-2) \cdots \cdots(2 m-k+1)}{(2 m+1) 2 m(2 m-1) \cdots(2 m-k+2)}\right) \\
& =N\left(\frac{2 m-k+2}{2 m+1}-\frac{2 m-k+1}{2 m+1}\right), \quad \text { if } n=2 m+1 \\
& =\frac{N}{n} .
\end{aligned}
$$

Finally, we have

$$
L^{0} \varphi\left(x_{1}, \ldots, x_{n}\right)=\frac{c^{2}}{n} \Delta \varphi\left(x_{1}, \ldots, x_{n}\right)
$$

Lemma is proved.

Proof of Theorem 3.1. In Lemma 3.3, we proved that $L^{\varepsilon} \varphi^{\varepsilon} \Rightarrow L^{0} \varphi$ at the class of functions $C_{0}^{\infty}\left(\mathbf{R}^{n} \times \Theta\right)$ when $\varepsilon \rightarrow 0$. To prove the weak convergence of the process, we should show relative compactness of the family $\left(\xi_{t}^{\varepsilon}, \theta_{t}^{\varepsilon}\right)$ in $\mathbf{D}_{\mathbf{R}^{n} \times \Theta}[0, \infty)$. To do this, we use the methods proposed in [15, 18, 19]. Let us formulate Corollary 6.1 from [15] (see also Theorem 6.4 in [15]) as Lemma 3.4.

Lemma 3.4. Let the generators $L^{\varepsilon}, \varepsilon>0$ satisfy the inequalities

$$
\left|L^{\varepsilon} \varphi(u)\right|<C_{\varphi}
$$

for any real-valued nonnegative function $\varphi \in C_{0}^{\infty}\left(\mathbf{R}^{n} \times \Theta\right)$, where the constant $C_{\varphi}$ depends on the norm of $\varphi$, and for $\varphi_{0}(u)=\sqrt{1+u^{2}}$,

$$
L^{\varepsilon} \varphi_{0}(u) \leq C_{l} \varphi_{0}(u), \quad|u| \leq l,
$$

where the constant $C_{l}$ depends on the function $\varphi_{0}$, but does not depend on $\varepsilon>0$.

Then, the family $\left(\xi_{t}^{\varepsilon}, \theta_{t}^{\varepsilon}\right), t \geq 0, \varepsilon>0$ is relatively compact in $\mathbf{D}_{\mathbf{R}^{n} \times \Theta}[0, \infty)$.

Let us write the action of the generator (2.3) at the test function $\varphi^{\varepsilon}\left(x_{1}, \ldots, x_{n} ; \theta\right)=$ $\varphi\left(x_{1}, \ldots, x_{n}\right)+\varepsilon \varphi_{1}\left(x_{1}, \ldots, x_{n} ; \theta\right)$, where $\varphi_{1}\left(x_{1}, \ldots, x_{n} ; \theta\right)=-c R_{0} S(\theta) \varphi\left(x_{1}, \ldots, x_{n}\right)$.

We have

$$
\begin{aligned}
L^{\varepsilon} \varphi^{\varepsilon}\left(x_{1}, \ldots, x_{n} ; \theta\right)= & \varepsilon^{-2} Q \varphi\left(x_{1}, \ldots, x_{n}\right)+\varepsilon^{-1}\left[Q \varphi_{1}+c S(\theta) \varphi\left(x_{1}, \ldots, x_{n}\right)\right] \\
& +c S(\theta) \varphi_{1}\left(x_{1}, \ldots, x_{n} ; \theta\right) .
\end{aligned}
$$


It follows from (3.7) that the first two terms equal to 0 . Let us estimate the following last term:

$$
\begin{aligned}
& c S(\theta) \varphi_{1}\left(x_{1}, \ldots, x_{n} ; \theta\right)=c^{2} S(\theta) R_{0} S(\theta) \varphi\left(x_{1}, \ldots, x_{n}\right)=c^{2} S^{2}(\theta) \varphi\left(x_{1}, \ldots, x_{n}\right) \\
& =c^{2}\left[\cos ^{2} \theta_{1} \frac{\partial^{2}}{\partial x_{1}^{2}}+\sin ^{2} \theta_{1} \cos ^{2} \theta_{2} \frac{\partial^{2}}{\partial x_{2}^{2}}\right. \\
& +\cdots+\sin ^{2} \theta_{1} \cdots \sin ^{2} \theta_{n-2} \cos ^{2} \theta_{n-1} \frac{\partial^{2}}{\partial x_{n-1}^{2}} \\
& +\sin ^{2} \theta_{1} \cdots \sin ^{2} \theta_{n-2} \sin ^{2} \theta_{n-1} \frac{\partial^{2}}{\partial x_{n}^{2}}+ \\
& \left\{\sin \theta_{1} \cos \theta_{1} \cos \theta_{2} \times \frac{\partial^{2}}{\partial x_{1} \partial x_{2}}+\cdots+\sin ^{2} \theta_{1} \cdots \sin ^{2} \theta_{n-2}\right. \\
& \left.\left.\times \sin \theta_{n-1} \times \cos \theta_{n-1} \frac{\partial^{2}}{\partial x_{n-1} \partial x_{n}}\right\}\right] \varphi\left(x_{1}, \ldots, x_{n}\right) \leq C_{1, \varphi},
\end{aligned}
$$

as soon as all the constants, functions, and their derivatives are bounded.

We also have from (3.7)

$$
L^{\varepsilon} \varphi^{\varepsilon}=L^{\varepsilon} \varphi+\varepsilon L^{\varepsilon} \varphi_{1}=L^{\varepsilon} \varphi+\varepsilon L^{\varepsilon} R_{0} S(\theta) \varphi
$$

Thus,

$$
L^{\varepsilon} \varphi=L^{\varepsilon} \varphi^{\varepsilon}-\varepsilon c L^{\varepsilon} R_{0} S(\theta) \varphi \leq C_{1, \varphi}-\varepsilon C_{2, \varphi}<C_{\varphi},
$$

for small $\varepsilon$.

To prove the second condition, it's enough to use the properties of the function $\varphi_{0}(u)=$ $\sqrt{1+u^{2}}$, namely:

$$
\left|\varphi_{0}^{\prime}(u)\right| \leq 1 \leq \varphi_{0}(u), \quad\left|\varphi_{0}^{\prime \prime}(u)\right| \leq \varphi_{0}(u) .
$$

So, the proof of the second condition is similar to the previous reasoning.

Thus, the family $\left(\xi_{t}^{\varepsilon}, \theta_{t}^{\varepsilon}\right)$ is relatively compact in $\mathbf{D}_{\mathbf{R}^{n} \times \Theta}[0, \infty)$.

Now we may use the following theorem (Theorem 6.6 from [15]).

Theorem 3.5. Let random evolution with Markov switching $\left(\xi^{\varepsilon}(t), x^{\varepsilon}(t)\right) \in \mathbf{D}_{\mathbf{R}^{n} \times E}[0, \infty)$ satisfies the following conditions.

(C1) The family of processes $\left(\xi^{\varepsilon}(t), x^{\varepsilon}(t)\right), t \geq 0, \varepsilon>0$ is relatively compact.

(C2) There exists the family of test-functions $\varphi^{\varepsilon}(u, x) \in C_{0}^{3}\left(\mathbf{R}^{n} \times E\right)$, such that

$$
\lim _{\varepsilon \rightarrow 0} \varphi^{\varepsilon}(u, x)=\varphi(u)
$$


uniformly by $u, x$.

(C3) The following uniform convergence is true

$$
\lim _{\varepsilon \rightarrow 0} L^{\varepsilon} \varphi^{\varepsilon}(u, x)=L \varphi(u)
$$

uniformly by $u, x$.

The family $L^{\varepsilon} \varphi^{\varepsilon}, \varepsilon>0$ is uniformly bounded, moreover $L^{\varepsilon} \varphi^{\varepsilon}$ and $L \varphi$ belong to $C\left(\mathbf{R}^{n} \times E\right)$.

(C4) Convergence by probability of initial values

$$
\begin{aligned}
& \xi^{\varepsilon}(0) \longrightarrow \widehat{\xi}(0), \quad \varepsilon \longrightarrow 0 \\
& \sup _{\varepsilon>0} \mathbf{E}\left|\xi^{\varepsilon}(0)\right| \leq C<+\infty
\end{aligned}
$$

is true.

Then we have the weak convergence

$$
\xi^{\varepsilon}(t) \Longrightarrow \widehat{\xi}(t), \quad \varepsilon \longrightarrow 0
$$

According to Theorem 3.5, we may confirm the weak convergence in $\mathbf{D}_{\mathbf{R}^{n}}[0, \infty)$

$$
\xi_{t}^{\varepsilon} \Longrightarrow \xi_{t}^{0}
$$

Really, all the conditions are satisfied. Namely, the family of processes is relatively compact, the generators converge at the test functions belonging to the class $C_{0}^{\infty}\left(\mathbf{R}^{n} \times \Theta\right)$, initial conditions for the limit, and prelimit processes are equal.

Theorem is proved.

\section{Description of MNRE}

We study the same particle in $\mathbf{R}^{n}$, but its velocity is equal to $v(\theta)=c(\theta) \varepsilon^{-1}+c_{1}(\theta)$, where $\varepsilon \rightarrow 0(\varepsilon>0)$ is the small parameter, the functions $c(\theta), c_{1}(\theta)$ are bounded.

Definition 4.1. Markov nonsymmetrical random evolution (MNRE) is the process $\tilde{\xi}_{t}^{\varepsilon} \in \mathbf{R}^{n}$, given by

$$
\tilde{\xi}_{t}^{\varepsilon}:=x+\int_{0}^{t} v\left(\theta_{\tau}^{\varepsilon}\right) s\left(\theta_{\tau}^{\varepsilon}\right) d \tau
$$

Our aim is to prove the weak convergence of MNRE to a diffusion process with drift when $\varepsilon \rightarrow 0$. 
Two-component Markov process $\left(\tilde{\xi}_{t}^{\varepsilon}, \theta_{t}^{\varepsilon}\right)$ at the test functions $\varphi\left(x_{1}, \ldots, x_{n} ; \theta\right) \in C_{0}^{\infty}\left(\mathbf{R}^{n} \times\right.$ $\Theta$ ) is defined by a generator (see, e.g., [2])

$$
\begin{aligned}
L^{\varepsilon} \varphi\left(x_{1}, \ldots, x_{n} ; \theta\right):= & \lambda Q \varphi(\cdot ; \theta)+v(\theta) S(\theta) \varphi\left(x_{1}, \ldots, x_{n} ; \cdot\right) \\
= & \varepsilon^{-2} Q \varphi(\cdot ; \theta)+\varepsilon^{-1} c(\theta) S(\theta) \varphi\left(x_{1}, \ldots, x_{n} ; \cdot\right) \\
& +c_{1}(\theta) S(\theta) \varphi\left(x_{1}, \ldots, x_{n} ; \cdot\right)
\end{aligned}
$$

where

$$
S(\theta) \varphi\left(x_{1}, \ldots, x_{n} ; \cdot\right):=-(s(\theta), \nabla) \varphi\left(x_{1}, \ldots, x_{n} ; \cdot\right)
$$

An important condition that let us confirm weak convergence is balance condition

$$
\Pi c(\theta) S(\theta)=0 .
$$

Remark 4.2. This is the last condition that defines symmetry or nonsymmetry of the process. In case of MSRE, the balance condition (4.4) is true, and $c_{1}(\theta) \equiv 0$. In case of MNRE the absence of symmetry of the process is caused by the following condition:

$$
\Pi c_{1}(\theta) S(\theta)=(d, \nabla) \neq 0 \text {. }
$$

Example 4.3. Condition (4.4) can be satisfied for different functions $c(\theta)$. Namely, in case of $\operatorname{MSRE} c(\theta)=c=$ const. Then every term under the integral contains either $\int_{0}^{\pi} \sin ^{n} \theta \cos \theta d \theta=$ 0 or $\int_{0}^{2 \pi} \sin \theta d \theta=0$.

Another example is the function $c(\theta)=\sin \theta_{1}$. Really, we obtain the terms under the integral that are analogical to the previous ones. We note that the dimension of the space in this case should be more than 2 , because in $\mathbf{R}^{2}$ this function does not have symmetry.

Example 4.4. Condition (4.5) can be also satisfied for different functions $c_{1}(\theta)$. For example, in $\mathbf{R}^{2}$ for $c_{1}(\theta)=\sin \theta$ we obtain

$$
\frac{1}{2 \pi} \int_{0}^{2 \pi} \sin \theta\left[\cos \theta \frac{\partial}{\partial x_{1}}+\sin \theta \frac{\partial}{\partial x_{2}}\right] d \theta=\frac{1}{2} \frac{\partial}{\partial x_{2}}
$$

Another example is the following function in $\mathbf{R}^{n}$ :

$$
c_{1}(\theta)= \begin{cases}c_{1}, & \theta_{n-1} \in[\pi, 2 \pi), \\ 0, & \theta_{n-1} \in[0, \pi) .\end{cases}
$$

Again, all terms under the integral, except the last one, contain $\int_{0}^{\pi} \sin ^{n} \theta \cos \theta d \theta=0$, thus only one term is not trivial as

$$
\frac{1}{N} c_{1} \int_{0}^{\pi} \cdots \int_{0}^{\pi} \int_{\pi}^{2 \pi} \sin ^{n-1} \theta_{1} \sin ^{n-2} \theta_{2} \cdots \sin ^{2} \theta_{n-1} \sin \theta_{n-1} d \theta_{1} \cdots d \theta_{n-1} .
$$


By simple calculations, we see

$$
\Pi c_{1}(\theta) S(\theta)= \begin{cases}-\frac{c_{1}}{2} \frac{3 \cdot 5 \cdots(n-2)}{2 \cdot 4 \cdots(n-1)} \frac{\partial}{\partial x_{n}}, & n=2 m+1 \\ -\frac{c_{1}}{\pi}[1 \cdot 2 \cdots(n-2)] \frac{\partial}{\partial x_{n}}, & n=2 m .\end{cases}
$$

We obviously have a wide range of functions that preserve or, on the contrary, do not preserve symmetry. So, we can define the velocity of random evolution in different ways, depending on possible applications.

\section{Main Result for MNRE}

Theorem 5.1. MNRE $\tilde{\xi}_{t}^{\varepsilon}$, converges weakly to the process $\tilde{\xi}_{t}^{0}$ when $\varepsilon \rightarrow 0$ as

$$
\tilde{\xi}_{t}^{\varepsilon} \Longrightarrow \tilde{\xi}_{t}^{0}
$$

The limit process $\widetilde{\xi}_{t}^{0} \in \mathbf{R}^{n}$ is defined by a generator

$$
L^{0} \varphi\left(x_{1}, \ldots, x_{n}\right)=(d, \nabla) \varphi\left(x_{1}, \ldots, x_{n}\right)+\left(\sigma^{2}, \Delta\right) \varphi\left(x_{1}, \ldots, x_{n}\right)
$$

where $\Delta \varphi\left(x_{1}, \ldots, x_{n}\right):=\left(\left(\partial^{2} / \partial x_{1}^{2}\right)+\cdots+\left(\partial^{2} / \partial x_{n}^{2}\right)\right) \varphi\left(x_{1}, \ldots, x_{n}\right)$,

$$
\begin{aligned}
& (d, \nabla):=-\frac{1}{N} \int_{S_{n}} c_{1}(\theta)(s(\theta), \nabla) \mu(d \theta) \\
& \left(\sigma^{2}, \Delta\right):=\frac{1}{N} \int_{S_{n}} c^{2}(\theta)(s(\theta), \nabla)^{2} \mu(d \theta) .
\end{aligned}
$$

We need the following Lemma to prove the Theorem.

Lemma 5.2. At the perturbed test functions

$$
\varphi^{\varepsilon}\left(x_{1}, \ldots, x_{n} ; \theta\right)=\varphi\left(x_{1}, \ldots, x_{n}\right)+\varepsilon \varphi_{1}\left(x_{1}, \ldots, x_{n} ; \theta\right)+\varepsilon^{2} \varphi_{2}\left(x_{1}, \ldots, x_{n} ; \theta\right),
$$

having bounded derivatives of any degree and compact support, the operator $L^{\varepsilon}$ has the following asymptotic representation:

$$
\begin{array}{r}
L^{\varepsilon} \varphi^{\varepsilon}\left(x_{1}, \ldots, x_{n} ; \theta\right)=L^{0} \varphi\left(x_{1}, \ldots, x_{n}\right)+R^{\varepsilon}(\theta) \varphi(x), \\
\left|R^{\varepsilon}(\theta) \varphi(x)\right| \longrightarrow 0, \quad \varepsilon \longrightarrow 0, \varphi(x) \in C_{0}^{\infty}\left(\mathbf{R}^{n}\right),
\end{array}
$$


where $L^{0}$ is defined in (5.2), $\varphi_{1}\left(x_{1}, \ldots, x_{n} ; \theta\right), \varphi_{2}\left(x_{1}, \ldots, x_{n} ; \theta\right)$, and $R^{\varepsilon}(\theta) \varphi(x)$ are defined by the following correlations:

$$
\begin{gathered}
L^{0} \Pi=-\Pi c(\theta) S(\theta) R_{0} c(\theta) S(\theta) \Pi+\Pi c_{1}(\theta) S(\theta) \Pi \varphi \\
\varphi_{1}=-R_{0} c(\theta) S(\theta) \varphi \\
\varphi_{2}=R_{0} c(\theta) S(\theta) R_{0} c(\theta) S(\theta) \varphi \\
R^{\varepsilon}(\theta) \varphi=\left\{\varepsilon\left[c(\theta) S(\theta) R_{0} c(\theta) S(\theta) R_{0} c(\theta) S(\theta)+c_{1}(\theta) S(\theta) R_{0} c(\theta) S(\theta)\right]\right. \\
\left.+\varepsilon^{2} c_{1}(\theta) S(\theta) R_{0} c(\theta) S(\theta) R_{0} c(\theta) S(\theta)\right\} \varphi .
\end{gathered}
$$

Proof. We solve singular perturbation problem for the operator (4.2). Using the view of testfunctions (5.4), we have

$$
\begin{aligned}
L^{\varepsilon} \varphi^{\varepsilon}\left(x_{1}, \ldots, x_{n} ; \theta\right)= & {\left[\varepsilon^{-2} Q+\varepsilon^{-1} c(\theta) S(\theta)+c_{1}(\theta) S(\theta)\right]\left[\varphi+\varepsilon \varphi_{1}+\varepsilon^{2} \varphi_{2}\right] } \\
= & \varepsilon^{-2} Q \varphi+\varepsilon^{-1}\left[Q \varphi_{1}+c(\theta) S(\theta) \varphi\right]+\left[Q \varphi_{2}+c(\theta) S(\theta) \varphi_{1}+c_{1}(\theta) S(\theta) \varphi\right] \\
& +\varepsilon\left[c(\theta) S(\theta) \varphi_{2}+c_{1}(\theta) S(\theta) \varphi_{1}\right]+\varepsilon^{2} c_{1}(\theta) S(\theta) \varphi_{2} .
\end{aligned}
$$

Thus, we obtain the following equations:

$$
\begin{gathered}
Q \varphi=0, \\
Q \varphi_{1}+c(\theta) S(\theta) \varphi=0, \\
L^{0} \varphi=Q \varphi_{2}+c(\theta) S(\theta) \varphi_{1}+c_{1}(\theta) S(\theta) \varphi, \\
R^{\varepsilon} \varphi(\theta)=\varepsilon\left[c(\theta) S(\theta) \varphi_{2}+c_{1}(\theta) S(\theta) \varphi_{1}\right]+\varepsilon^{2} c_{1}(\theta) S(\theta) \varphi_{2} .
\end{gathered}
$$

According to the first one, $\varphi\left(x_{1}, \ldots, x_{n}\right)$ belongs to the null space of $Q$. From the balance condition (4.4) we see that $c(\theta) S(\theta) \varphi$ belongs to the range of $Q$, thus from the second equation of (5.8) we have

$$
\varphi_{1}=-R_{0} c(\theta) S(\theta) \varphi
$$

By substitution into the third equation and using the solvability condition, we obtain:

$$
L^{0} \Pi \varphi+\Pi c(\theta) S(\theta) R_{0} c(\theta) S(\theta) \Pi \varphi-\Pi c_{1}(\theta) S(\theta) \Pi \varphi=0 .
$$


From the last equation, we have

$$
\begin{gathered}
R^{\varepsilon}(\theta) \varphi(x)=\left\{\varepsilon\left[c(\theta) S(\theta) R_{0} c(\theta) S(\theta) R_{0} c(\theta) S(\theta)+c_{1}(\theta) S(\theta) R_{0} c(\theta) S(\theta)\right]\right. \\
\left.+\varepsilon^{2} c_{1}(\theta) S(\theta) R_{0} c(\theta) S(\theta) R_{0} c(\theta) S(\theta)\right\} \varphi(x) \longrightarrow 0 \\
\text { when } \varepsilon \longrightarrow 0, \varphi(x) \in C_{0}^{\infty}\left(\mathbf{R}^{n}\right) .
\end{gathered}
$$

We calculate the operator $L^{0}$ by the formula (5.6) as

$$
\begin{aligned}
L^{0} \varphi & =\Pi c(\theta) S(\theta)(I-\Pi) c(\theta) S(\theta) \Pi \varphi+\Pi c_{1}(\theta) S(\theta) \Pi \varphi \\
& =\Pi c^{2}(\theta) S^{2}(\theta) \Pi \varphi-\Pi c(\theta) S(\theta) \Pi c(\theta) S(\theta) \Pi \varphi+\Pi c_{1}(\theta) S(\theta) \Pi \varphi .
\end{aligned}
$$

The second term equals 0 by the balance condition (4.4), the last one is not equal to 0 by (4.5). Thus, we finally have

$$
L^{0}=\Pi c^{2}(\theta) S^{2}(\theta)+\Pi c_{1}(\theta) S(\theta)
$$

Using the view of $S(\theta)$, we may write

$$
\begin{aligned}
\Pi c_{1}(\theta) S(\theta) & =-\frac{1}{N} \int_{S_{n}} c_{1}(\theta)(s(\theta), \nabla) \mu(d \theta)=:(d, \nabla), \\
\Pi c^{2}(\theta) S^{2}(\theta) & =\frac{1}{N} \int_{S_{n}} c^{2}(\theta)(s(\theta), \nabla)^{2} \mu(d \theta)=:\left(\sigma^{2}, \Delta\right) .
\end{aligned}
$$

Lemma is proved.

Proof of Theorem 5.1. We proved in Lemma 5.2 that $L^{\varepsilon} \varphi^{\varepsilon} \Rightarrow L^{0} \varphi$ at the class of test functions $C_{0}^{\infty}\left(\mathbf{R}^{n} \times \Theta\right)$, when $\varepsilon \rightarrow 0$. To prove the weak convergence, we should show relative compactness of the family of processes $\left(\tilde{\xi}_{t}^{\varepsilon}, \theta_{t}^{\varepsilon}\right)$ in $\mathbf{D}_{\mathbf{R}^{n} \times \Theta}[0, \infty)$. To do this, we use Lemma 3.4.

We have the following view of the operator (4.2) at the test function $\varphi^{\varepsilon}\left(x_{1}, \ldots, x_{n} ; \theta\right)=$ $\varphi\left(x_{1}, \ldots, x_{n}\right)+\varepsilon \varphi_{1}\left(x_{1}, \ldots, x_{n} ; \theta\right)$, where $\varphi_{1}\left(x_{1}, \ldots, x_{n} ; \theta\right)=-R_{0} c(\theta) S(\theta) \varphi\left(x_{1}, \ldots, x_{n}\right)$ :

$$
\begin{aligned}
L^{\varepsilon} \varphi^{\varepsilon}\left(x_{1}, \ldots, x_{n} ; \theta\right)= & \varepsilon^{-2} Q \varphi\left(x_{1}, \ldots, x_{n}\right)+\varepsilon^{-1}\left[Q \varphi_{1}+c(\theta) S(\theta) \varphi\left(x_{1}, \ldots, x_{n}\right)\right] \\
& +\left[c(\theta) S(\theta) \varphi_{1}\left(x_{1}, \ldots, x_{n} ; \theta\right)+c_{1}(\theta) S(\theta) \varphi\left(x_{1}, \ldots, x_{n} ; \theta\right)\right] \\
& +\varepsilon c_{1}(\theta) S(\theta) \varphi_{1}\left(x_{1}, \ldots, x_{n} ; \theta\right) .
\end{aligned}
$$


It follows from (5.8) that the first two terms equal to 0 . Let us estimate the following third term:

$$
\begin{gathered}
c(\theta) S(\theta) \varphi_{1}\left(x_{1}, \ldots, x_{n} ; \theta\right)+c_{1}(\theta) S(\theta) \varphi\left(x_{1}, \ldots, x_{n} ; \theta\right) \\
=c(\theta) S(\theta) R_{0} c(\theta) S(\theta) \varphi\left(x_{1}, \ldots, x_{n}\right)+c_{1}(\theta) S(\theta) \varphi\left(x_{1}, \ldots, x_{n} ; \theta\right) \\
=c^{2}(\theta) S^{2}(\theta) \varphi\left(x_{1}, \ldots, x_{n}\right)+c_{1}(\theta) S(\theta) \varphi\left(x_{1}, \ldots, x_{n} ; \theta\right) \\
=c^{2}(\theta)\left[\cos ^{2} \theta_{1} \frac{\partial^{2}}{\partial x_{1}^{2}}+\sin ^{2} \theta_{1} \cos ^{2} \theta_{2} \frac{\partial^{2}}{\partial x_{2}^{2}}\right. \\
+\cdots+\sin ^{2} \theta_{1} \cdots \sin ^{2} \theta_{n-2} \cos ^{2} \theta_{n-1} \frac{\partial^{2}}{\partial x_{n-1}^{2}}+\sin ^{2} \theta_{1} \cdots \sin ^{2} \theta_{n-2} \sin ^{2} \theta_{n-1} \frac{\partial^{2}}{\partial x_{n}^{2}} \\
+\left\{\sin \theta_{1} \cos \theta_{1} \cos \theta_{2} \frac{\partial^{2}}{\partial x_{1} \partial x_{2}}+\cdots+\sin ^{2} \theta_{1} \cdots \sin ^{2} \theta_{n-2} \sin \theta_{n-1}\right. \\
\left.\left.\quad \times \cos \theta_{n-1} \frac{\partial^{2}}{\partial x_{n-1} \partial x_{n}}\right\}\right] \varphi\left(x_{1}, \ldots, x_{n}\right)+c_{1}(\theta) \\
{\left[\cos \theta_{1} \frac{\partial}{\partial x_{1}}+\sin \theta_{1} \cos \theta_{2} \frac{\partial}{\partial x_{2}}+\cdots+\sin \theta_{1} \ldots \sin \theta_{n-2} \cos \theta_{n-1} \frac{\partial}{\partial x_{n-1}}\right.} \\
\left.+\sin \theta_{1} \cdots \sin \theta_{n-2} \sin \theta_{n-1} \frac{\partial}{\partial x_{n}}\right] \varphi\left(x_{1}, \ldots, x_{n}\right) \leq C_{1, \varphi}
\end{gathered}
$$

as soon as all the constants, functions, and their derivatives are bounded.

The last term may be estimated analogically.

From (5.8) we also have

$$
L^{\varepsilon} \varphi^{\varepsilon}=L^{\varepsilon} \varphi+\varepsilon L^{\varepsilon} \varphi_{1}=L^{\varepsilon} \varphi+\varepsilon c L^{\varepsilon} R_{0} \theta S(\theta) \varphi
$$

Thus,

$$
L^{\varepsilon} \varphi=L^{\varepsilon} \varphi^{\varepsilon}-\varepsilon c L^{\varepsilon} R_{0} S(\theta) \varphi \leq C_{1, \varphi}-\varepsilon C_{2, \varphi}<C_{\varphi}
$$

for $\varepsilon$ that is small enough.

To prove the second condition of Lemma 3.4, we use the following properties of the function $\varphi_{0}(u)=\sqrt{1+u^{2}}$ :

$$
\left|\varphi_{0}^{\prime}(u)\right| \leq 1 \leq \varphi_{0}(u), \quad\left|\varphi_{0}^{\prime \prime}(u)\right| \leq \varphi_{0}(u) .
$$

We can see again that the proof of the second condition is similar to the previous reasoning.

Thus, the family of the processes $\left(\tilde{\xi}_{t}^{\varepsilon}, \theta_{t}^{\varepsilon}\right)$ is relatively compact in $\mathbf{D}_{\mathbf{R}^{n} \times \Theta}[0, \infty)$. 
Using Theorem 3.5, we confirm the following weak convergence in $\mathbf{D}_{\mathbf{R}^{n}}[0, \infty)$ :

$$
\widetilde{\xi}_{t}^{\varepsilon} \Longrightarrow \widetilde{\xi}_{t}^{0}
$$

Really, all the conditions are satisfied. Namely, the family of processes is relatively compact, the generators at the test functions belonging to the class $C_{0}^{\infty}\left(\mathbf{R}^{n} \times \Theta\right)$ converge, and initial conditions for the limit and prelimit processes are equal.

Theorem is proved.

Example 5.3. Let us consider one more variant of evolution in $\mathbf{R}^{2}$.

Let

$$
\begin{aligned}
& c(\theta)= \begin{cases}1, & \theta=0, \\
1, & \theta=\pi,\end{cases} \\
& c_{1}(\theta)=1, \quad \theta=\frac{\pi}{2} .
\end{aligned}
$$

In other cases both functions equal to 0 .

The balance condition (4.4) is true for $c(\theta)$, on the contrary, condition (4.5) is true for $c_{1}(\theta)$.

The limit generator (5.2) has the view

$$
L^{0} \varphi\left(x_{1}, x_{2}\right)=\frac{1}{2 \pi} \frac{\partial}{\partial x_{2}} \varphi\left(x_{1}, x_{2}\right)+\frac{1}{\pi} \frac{\partial^{2}}{\partial x_{1}^{2}} \varphi\left(x_{1}, x_{2}\right)
$$

Thus, the limit process has two parts: the drift with velocity $1 / 2 \pi$ in direction of $x_{2}$ coordinate and diffusion part in one-dimensional subspace, corresponding to $x_{1}$ coordinate that is similar to the limit process described in Kac model [1].

\section{List of Symbols}

$$
\begin{aligned}
& c, c(\theta), c_{1}(\theta): \\
& C_{0}^{\infty}\left(\mathbf{R}^{n} \times \Theta\right): \\
& C_{\varphi}: \\
& d: \\
& \mathbf{D}_{\mathbf{R}^{n} \times \Theta}[0, \infty): \\
& L^{\varepsilon}: \\
& L^{0}: \\
& N: \\
& n: \\
& Q:
\end{aligned}
$$

the components of velocity of random evolution's motion the space of bounded continuous functions on $\mathbf{R}^{n} \times \Theta$ having continuous derivatives of all orders vanishing at infinity constant depending on the norm of function $\varphi$ parameter of drift the space of $\mathbf{R}^{n} \times \Theta$-valued right continuous functions having left limits (cadlag), defined on $\mathbf{R}_{+}$ the generator of random evolution the generator of limit process volume of $S_{n}$ dimension of space the generator of switching Poisson process 


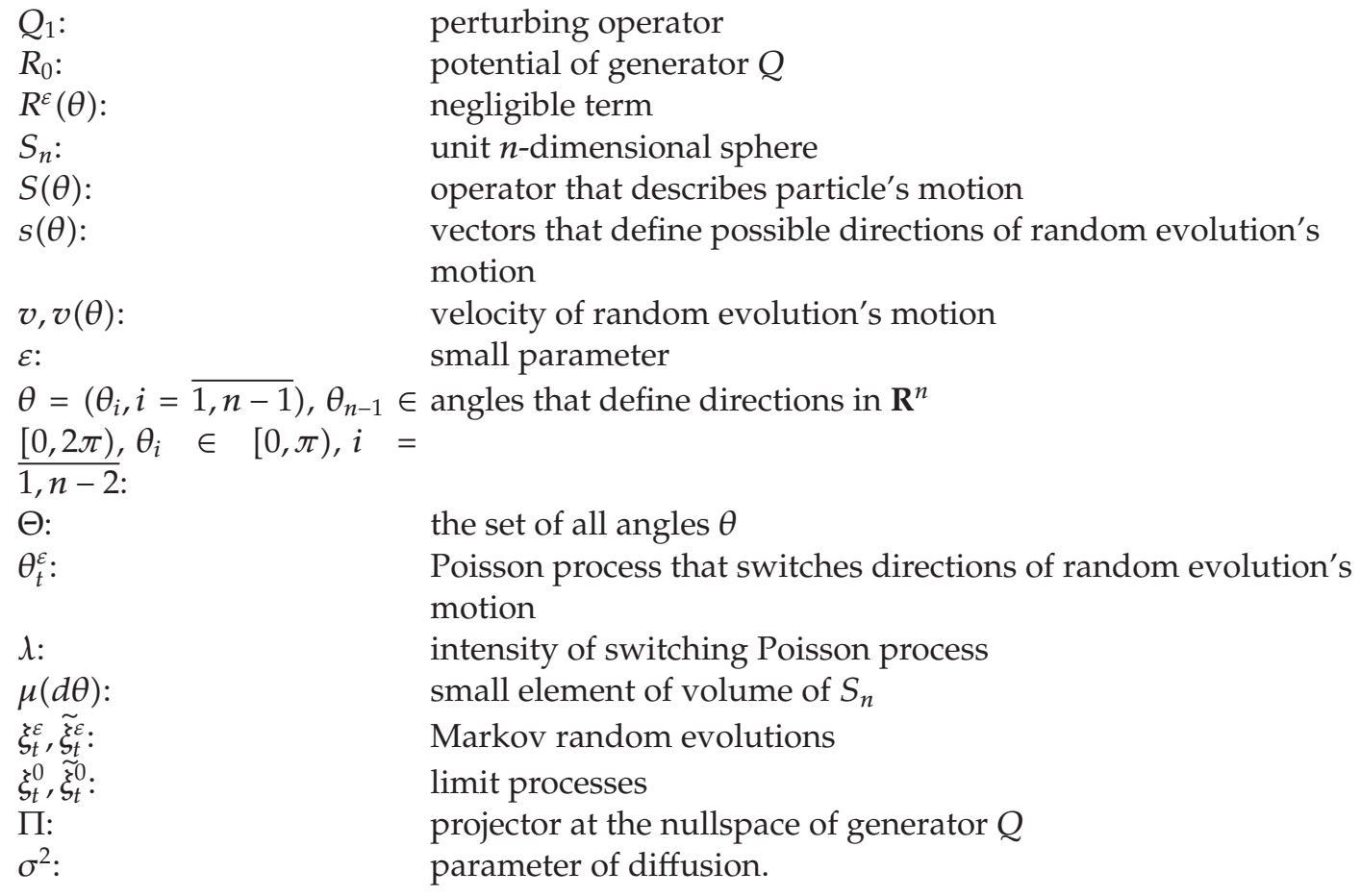

\section{References}

[1] M. Kac, "A stochastic model related to the telegrapher's equation," The Rocky Mountain Journal of Mathematics, vol. 4, pp. 497-509, 1974.

[2] M. A. Pinsky, Lectures on Random Evolution, World Scientific Publishing, Singapore, 1991.

[3] E. Orsingher, "Bessel functions of third order and the distribution of cyclic planar motions with three directions," Stochastics and Stochastics Reports, vol. 74, no. 3-4, pp. 617-631, 2002.

[4] E. Orsingher and A. M. Sommella, "A cyclic random motion in $\mathbf{R}^{3}$ with four directions and finite velocity," Stochastics and Stochastics Reports, vol. 76, no. 2, pp. 113-133, 2004.

[5] A. D. Kolesnik and A. F. Turbin, "Infinitesimal hyperbolic operator of Markovian random evolutions," Doklady Akademii Nauk Ukrainskoj, vol. 1, pp. 11-14, 1991 (Russian).

[6] A. D. Kolesnik and A. F. Turbin, "Symmetric random evolutions in $\mathbf{R}^{2}$," Doklady Akademii Nauk Ukrainskoj, vol. 2, pp. 10-11, 1990 (Russian).

[7] I. V. Samoilenko, "Markovian random evolution in $\mathbf{R}^{n}$," Random Operators and Stochastic Equations, vol. 9, no. 2, pp. 139-160, 2001.

[8] A. D. Kolesnik, "Weak convergence of the distributions of Markovian random evolutions in two and three dimensions," Buletinul Academiei de Ştiinţe a Republicii Moldova. Matematica, no. 3, pp. 41-52, 2003.

[9] A. D. Kolesnik, "Weak convergence of a planar random evolution to the Wiener process," Journal of Theoretical Probability, vol. 14, no. 2, pp. 485-494, 2001.

[10] A. A. Borovkov and K. A. Borovkov, Asymptotic Analysis of Random Walks, vol. 118 of Encyclopedia of Mathematics and its Applications, Cambridge University Press, Cambridge, UK, 2008.

[11] K. Borovkov, "On random walks with jumps scaled by cumulative sums of random variables," Statistics \& Probability Letters, vol. 35, no. 4, pp. 409-416, 1997.

[12] S. Foss, T. Konstantopoulos, and S. Zachary, "Discrete and continuous time modulated random walks with heavy-tailed increments," Journal of Theoretical Probability, vol. 20, no. 3, pp. 581-612, 2007.

[13] S. Zakhari and S. G. Foss, "On the exact asymptotics of the maximum of a random walk with increments in a class of light-tailed distributions," Siberian Mathematical Journal, vol. 47, no. 6, pp. 1034-1041, 2006. 
[14] V. V. Anisimov, Switching Processes in Queueing Models, Applied Stochastic Methods Series, ISTE, London, UK, John Wiley \& Sons, Hoboken, NJ, USA, 2008.

[15] V. S. Koroliuk and N. Limnios, Stochastic Systems in Merging Phase Space, World Scientific Publishing, Singapore, 2005.

[16] V. V. Anīsīmov, "Convergence of accumulation processes with switchings," Teorīya Imovīrnoster̆ ta Matematichna Statistika, no. 63, pp. 3-12, 2000, translation in Theory of Probability and Mathematical Statistics no. 63, 1-11, 2001-2002.

[17] I. V. Samoilenko, "Convergence of an impulsive storage process with jump switchings," Ukrä̈ni Matematicni Zhurnal, vol. 60, no. 9, pp. 1282-1286, 2008, translation in Ukrainian Mathematical Journal, vol. 60, no. 9, 1492-1497, 2008.

[18] S. N. Ethier and T. G. Kurtz, Markov Processes: Characterization and Convergence, Wiley Series in Probability and Mathematical Statistics: Probability and Mathematical Statistics, John Wiley \& Sons, New York, NY, USA, 1986.

[19] D. W. Stroock and S. R. S. Varadhan, Multidimensional Diffusion Processes, vol. 233 of Fundamental Principles of Mathematical Sciences, Springer, Berlin, Germany, 1979. 


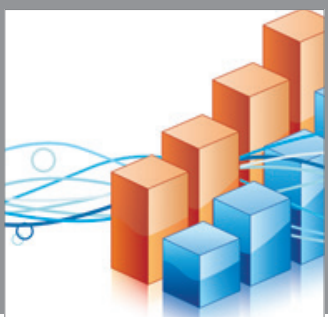

Advances in

Operations Research

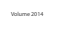

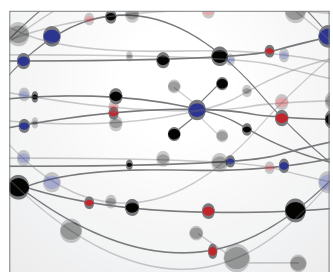

\section{The Scientific} World Journal
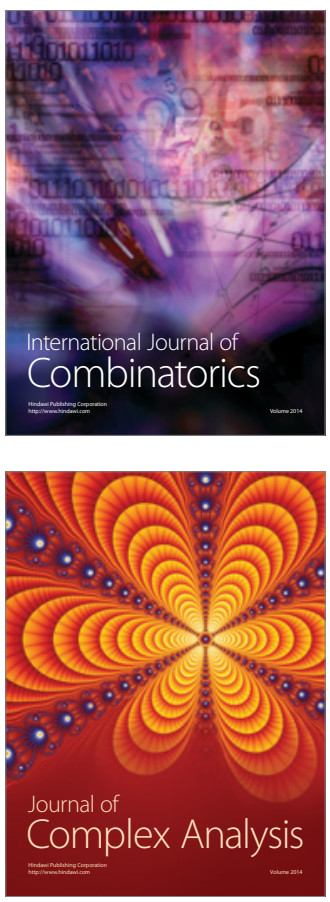

International Journal of

Mathematics and

Mathematical

Sciences
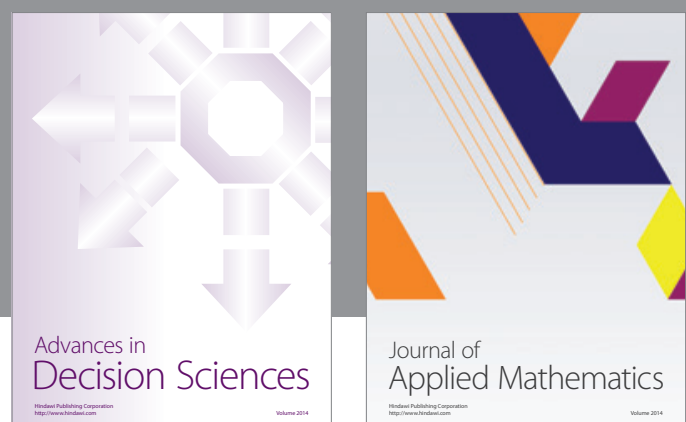

Journal of

Applied Mathematics
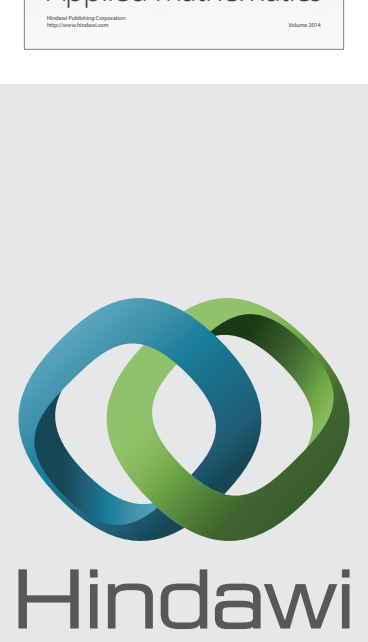

Submit your manuscripts at http://www.hindawi.com
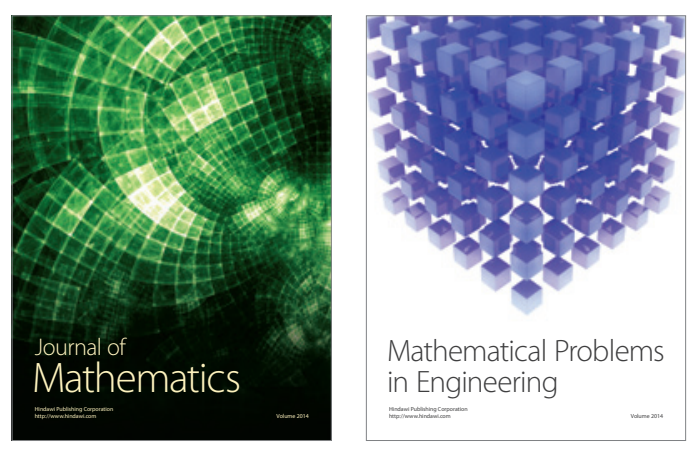

Mathematical Problems in Engineering
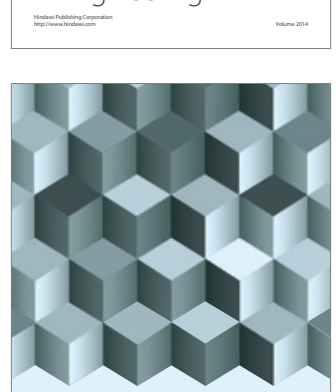

Journal of

Function Spaces
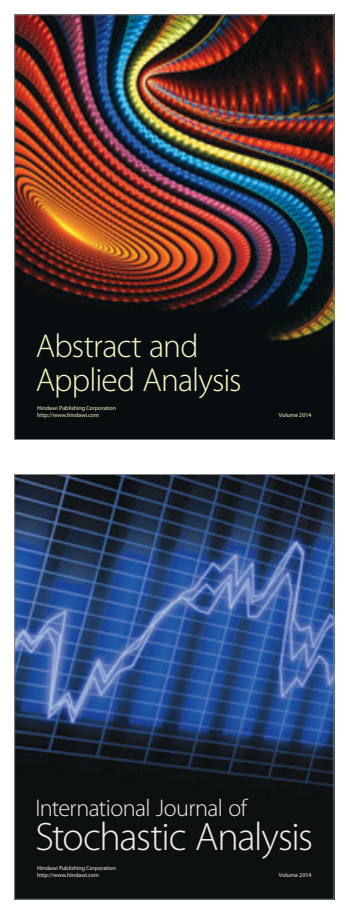

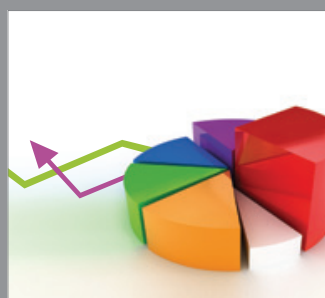

ournal of

Probability and Statistics

Promensencen
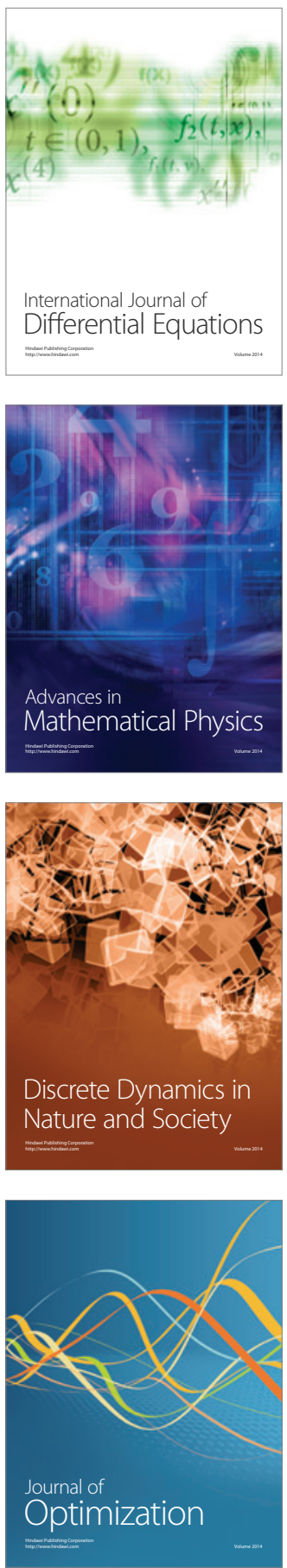